\title{
Simulation of Runaway Electron Generation During Plasma Shutdown by Impurity Injection in ITER
}

\author{
T. Fehér ${ }^{1,2}$, H. M. Smith ${ }^{1,3}$, T. Fülöp ${ }^{2}$ and K. Gál ${ }^{4}$ \\ ${ }^{1}$ Max-Planck-Institut für Plasmaphysik, Greifswald, Germany \\ ${ }^{2}$ Department of Applied Physics, Nuclear Engineering, Chalmers University of \\ Technology and Euratom-VR Association, Göteborg, Sweden \\ ${ }^{3}$ Max-Planck-Institut für Sonnensystemforschung, Katlenburg-Lindau, Germany \\ ${ }^{4}$ KFKI Research Institute for Particle and Nuclear Physics, Budapest, Hungary \\ E-mail: tamas.bela.feher@ipp.mpg.de
}

\begin{abstract}
Disruptions in a large tokamak can cause serious damage to the device and should be avoided or mitigated. Massive gas or killer pellet injection are possible ways to obtain a controlled fast plasma shutdown before a natural disruption occurs. In this work, plasma shutdown scenarios with different types of impurities are studied for an ITER-like plasma. Plasma cooling, runaway generation and the associated electric field diffusion are calculated with a 1D-code taking the Dreicer, hot-tail and avalanche runaway generation processes into account. Thin, radially localised sheets with high temperature can be created after the thermal quench, and the Dreicer and avalanche processes produce a high runaway current inside these sheets. At high impurity concentration the Dreicer process is suppressed but hot-tail runaways are created. Favourable thermal and current quench times can be achieved with a mixture of deuterium and neon or argon. However, to prevent the avalanche process from creating a significant runaway current fraction, it is found to be necessary to include runaway losses in the model.
\end{abstract}




\section{Introduction}

Plasma-terminating disruptions are one of the crucial problems that ITER [1] and similar devices will face. The sudden loss of the thermal energy $(\sim 1$ GJ) and the subsequent rapid quench of the plasma current $(\geq 10 \mathrm{MA})$ is dangerous not only because of the prompt heat load during the thermal quench (of the order of $10^{4} \mathrm{MW} / \mathrm{m}^{2}$ for timescales of $\sim 1 \mathrm{~ms}$ in the divertor of ITER), but also because of the electromechanical stresses induced by the current quench. In some cases, the current quench can be accompanied by a vertical plasma displacement in which the current channel moves rapidly towards the wall, inducing asymmetric halo currents. These events would exert large forces on the vacuum vessel, and would lead to severe restrictions on the design of its components. Development of techniques to safely terminate the discharge and mitigate the destructive effects of disruptions is one of the most critical issues for ITER and other large-scale power-producing tokamaks.

Fast plasma shutdown by impurity injection could be a possible way to avoid disruption related damage [2]. Impurities cool down the plasma through isotropically distributed radiation, which reduces the high local heat loads on the first wall compared with a natural disruption. The large impulse transferred to the vessel and its components as an effect of poloidal halo currents can also be reduced, if the plasma becomes so cool that the current quench time is short enough (for ITER $\tau_{\mathrm{CQ}} \lesssim 0.5 \mathrm{~s}$ is required [3]). However, if the temperature is too low, very energetic electrons may be produced. As the temperature drops on the short time scale of the thermal quench, an electric field is induced which keeps the current constant. When the field is higher than a certain critical electric field $E_{\mathrm{c}}$, a population of electrons can be accelerated to relativistic speeds. These so-called runaway electrons (REs) can form high energy beams that may seriously damage the device upon impact. A cooling scenario should be chosen so that not only the current quench time is sufficiently short but also so that runaway production is avoided.

Experiments with massive gas injection $[4,5,6,7]$ and killer pellet injection $[8,9,10]$ show that fast plasma shutdown can be achieved by impurity injection. One aim of such experiments is to suppress RE generation by a suitable choice of the impurity materials and of the amount of impurities to be injected. However, extrapolations to reactor parameters are not straightforward. Simulations of killer pellet injection $[11,12,13]$ have been performed by coupling runaway models to pellet injection and impurity radiation models. Simulations of massive gas injection involving sophisticated fluid models $[14,15]$ have also been made, but the inclusion of runaway effects is in its early stages. Often simpler 0D simulations are used in order to model experimental results $[7,16]$.

The aim of this work is to investigate fast shutdown for an ITER-like plasma using different types of impurities, and to try to find scenarios suitable for runawayfree disruption mitigation. We explore in detail what effect different concentrations of injected neon and argon have on the resulting current quench time and on the number of runaway electrons in ITER-like plasmas. We also investigate to what extent 
the runaway generation can be suppressed by introducing magnetic perturbations or injecting additional deuterium in the plasma.

The simulations presented in this paper are performed with a $1 \mathrm{D}$ runaway code, which was initially presented in [17, 18] and developed further in [11, 19], where it was applied to impurity injection scenarios in JET-like plasmas. The code determines the temperature evolution by taking into account radiation, Ohmic heating, heat diffusion and collisions between different particle species. The process of how the impurities are injected into the plasma is not addressed in the present work. Instead, we use a predescribed impurity density profile, together with a sensitivity analysis investigating the effect of various impurity density profiles. The primary runaway processes included in this work are the Dreicer and hot-tail processes and a simple estimate for runaways created by Compton scattering with high energy gamma rays from the activated wall. These processes provide a seed population of runaways that is further amplified by the (secondary) avalanche mechanism. A similar model was used in [2], but without the Dreicer and hot-tail runaway electron mechanisms.

An example of an ITER simulation using the present model for a low argon fraction was presented in [20]. Here, we make a more extensive study and compare results for various cases with different fractions of impurities in the plasma, including cases with different deuterium-impurity mixture. In contrast to JET [20], where injection of a large amount of pure argon would suppress runaway generation due to the resulting high electron density, our results show that in ITER high impurity fractions alone are not expected to suppress the runaway generation. It is necessary to simultaneously inject a large amount of deuterium or at the same time have a sufficient level of magnetic perturbations.

The remainder of the paper is organized as follows. In Sect. II the runaway generation and loss processes included in the present model are discussed. The impurity induced plasma cooling and the corresponding evolution of the electric field is described in Sec. III. In Sec. IV we present results for disruption mitigation scenarios with different impurity species and in Sec. V we summarize our conclusions.

\section{Runaway electron generation and losses}

The accelerating force on the electrons due to the electric field is counteracted by the friction force due to collisions. Since the collision frequency decreases as $v^{-3}$ at high velocities, there is a critical velocity, $v_{\mathrm{c}}=v_{T} \sqrt{E_{D} / 2 E}$ above which the electrons are continuously accelerated until they reach relativistic speeds. Here, $E_{\mathrm{D}}=m_{\mathrm{e}}^{2} c^{3} /\left(e \tau T_{\mathrm{e}}\right)$ is the Dreicer field, and $\tau$ is the relativistic electron collision time $\tau=4 \pi \varepsilon_{0}^{2} m_{\mathrm{e}}^{2} c^{3} /\left(n_{\mathrm{e}} e^{4} \ln \Lambda\right)$. The runaway acceleration is possible when the electric field is larger than the critical value $E_{\mathrm{c}}=m_{\mathrm{e}} c /(e \tau)$.

For a precise description of the runaway electron phenomenon one would need to 
solve the kinetic equation

$$
\frac{\partial f}{\partial t}+\mathbf{v} \cdot \frac{\partial f}{\partial \mathbf{r}}-\frac{e \mathbf{E}}{m_{e}} \cdot \frac{\partial f}{\partial \mathbf{v}}=C(f)+S(f)
$$

where $C(f)$ is the (relativistic) Fokker-Planck collision operator and $S(f)$ is a source/sink term that describes close collisions and runaway loss mechanisms. A numerical solution of this problem [21] is computationally very expensive. Therefore, we will use estimates of the number of electrons that enter the runaway region of velocity space $\left(v>v_{c}(E)\right)$ due to different runaway mechanisms. Instead of modelling the velocity space dynamics for the electrons that are already inside the runaway region, we only consider their total density $n_{\text {run }}$. This density evolves due to the Dreicer, hottail, $\gamma$-ray Compton scattering and avalanche generation mechanisms, and due to radial diffusion caused by magnetic field fluctuations,

$$
\begin{aligned}
\frac{\partial n_{\text {run }}}{\partial t}= & \left(\frac{\partial n_{\text {run }}}{\partial t}\right)^{\text {Dreicer }}+\left(\frac{\partial n_{\text {run }}}{\partial t}\right)^{\text {hot-tail }}+\left(\frac{\partial n_{\text {run }}}{\partial t}\right)^{\gamma}+ \\
& +\left(\frac{\partial n_{\text {run }}}{\partial t}\right)^{\text {avalanche }}+\frac{1}{r} \frac{\partial}{\partial r} r D_{\mathrm{RR}} \frac{\partial n_{\text {run }}}{\partial r} .
\end{aligned}
$$

The radial diffusion coefficient is here given by the Rechester-Rosenbluth estimate [22] $D_{R R}=\pi q v_{\|} R(\delta B / B)^{2}$, where $q$ is the safety factor, $v_{\|} \simeq c$ is the parallel velocity, $R$ is the major radius and $\delta B / B$ is the normalized magnetic perturbation amplitude. In reality, the diffusion coefficient is lower due to the averaging effect of the large particle orbits [23]. Runaway electrons can also be lost from the plasma due to several other processes that are not included in this study, e.g. resonant interaction with waves [24]. The Dreicer mechanism produces runaways by velocity space diffusion into the runaway region due to small angle collisions. Assuming quasi-steady state for the electron distribution function the generation rate is [25]

$$
\left(\frac{d n_{\text {run }}}{d t}\right)^{\text {Dreicer }} \simeq \frac{n_{\mathrm{e}}}{\tau}\left(\frac{m_{\mathrm{e}} c^{2}}{2 T_{\mathrm{e}}}\right)^{3 / 2}\left(\frac{E_{\mathrm{D}}}{E}\right)^{3\left(1+Z_{\mathrm{eff}}\right) / 16} e^{-\frac{E_{\mathrm{D}}}{4 E}-\sqrt{\frac{\left(1+Z_{\mathrm{eff}}\right) E_{\mathrm{D}}}{E}}} .
$$

The hot-tail mechanism is efficient if the cooling rate is comparable with the collision frequency, so that the quasi-steady state approximation used to obtain Eq. (3) does not hold. Impurity radiation mainly cools the low energy part of the electron distribution, whereas the high energy part will be cooled by collisions with thermal electrons. At high energies the electrons have lower collision frequency, so they cannot thermalize as quickly as the low energy bulk of the distribution. For a short while, they are therefore left as an elevated hot-tail of the distribution function. If the critical velocity decreases rapidly, these electrons may end up inside the runaway region and become runaways.

In the present model, the Fokker-Planck equation for energetic electrons colliding with a Maxwellian bulk distribution of electrons with temperature $T$ is solved without taking the electric field into account. It is assumed that the electric field only plays the role of delineating the runaway region. For certain cooling types there exists analytical solutions for this problem $[26,19]$. However, the calculated temperature decay caused 
by radiating impurities does not in general follow these special cases. Therefore, we solve the following kinetic equation numerically

$$
\frac{\partial f}{\partial t}=C(f)=\frac{e^{4} \ln \Lambda n}{8 \pi \epsilon_{0}^{2} m_{e}} \frac{1}{v^{2}} \frac{\partial}{\partial v}\left[v^{2} G\left(v / v_{T}\right)\left(\frac{1}{T} f(v)+\frac{1}{m_{e} v} \frac{\partial f(v)}{\partial v}\right)\right],
$$

where $\mathrm{G}$ is the Chandrasekhar function, and $\ln \Lambda=14.9-0.5 \ln \left(n_{\mathrm{e}} / 10^{20}\right)+\ln \left(T_{\mathrm{e}}\right)$ is the Coulomb logarithm [27]. The kinetic equation is solved with a fully implicit finite differences method, and the boundary conditions are calculated from the analytical limits. Knowing the distribution function, the number of runaways can be estimated as the velocity space integral of $f$ over the runaway region [19]

$$
\left(\frac{d n_{\text {run }}}{d t}\right)^{\text {hot-tail }}=4 \pi \frac{d}{d t} \int_{v_{\mathrm{c}}}^{\infty}\left(v^{2}-v_{c}^{2}\right) f \mathrm{~d} v .
$$

The avalanche mechanism is caused by close collisions between runaways and thermal electrons, and results in an exponential growth of the runaway density, here modelled by the analytical interpolation formula derived by Rosenbluth and Putvinski [28]

$$
\begin{aligned}
\left(\frac{d n_{\mathrm{run}}}{d t}\right)^{\text {avalanche }} \simeq & n_{\mathrm{run}} \frac{E / E_{\mathrm{c}}-1}{\tau \ln \Lambda} \sqrt{\frac{\pi \varphi}{3\left(Z_{\mathrm{eff}}+5\right)}} \times \\
& \left(1-\frac{E_{\mathrm{c}}}{E}+\frac{4 \pi\left(Z_{\mathrm{eff}}+1\right)^{2}}{3 \varphi\left(Z_{\mathrm{eff}}+5\right)\left(E^{2} / E_{\mathrm{c}}^{2}+4 / \varphi^{2}-1\right)}\right)^{-1 / 2},
\end{aligned}
$$

where $\varphi=\left(1+1.46 \epsilon^{1 / 2}+1.72 \epsilon\right)^{-1}$ and $\epsilon=r / R$ denotes the inverse aspect ratio. The avalanche amplifies the seed population of runaways created by the primary processes (Dreicer and hot-tail), and is a very powerful runaway mechanism in tokamaks with large current.

In ITER, tritium decay and Compton scattering of $\gamma$-rays emitted by the activated wall are also possible primary runaway electron sources. The magnitude of the runaway electron generation rate from $\gamma$-ray Compton scattering is uncertain, because it depends on the activation of the wall. In most of the simulations presented here the other two primary runaway mechanisms already produce a significant runaway seed current. However, when the Dreicer generation is suppressed and the hot-tail generation is absent, the number of runaways produced by the Compton scattering process cannot be neglected. For simplicity, we assume that the runaway generation is constant in the whole plasma and can be calculated by the following formula $\left(\partial n_{\text {run }} / \partial t\right)^{\gamma}=\sigma \Gamma_{\gamma} n_{\mathrm{e} 0}$. Here $\Gamma_{\gamma} \approx 10^{18} \mathrm{~m}^{-2} \mathrm{~s}^{-1}$ is the gamma ray flux [29], $n_{\mathrm{e} 0}=10^{20} \mathrm{~m}^{-3}$ is the electron density, and it is assumed that the cross section $\sigma$ is 1 barn. With these values $\left(\partial n_{\text {run }} / \partial t\right)^{\gamma}=10^{10} \mathrm{~m}^{-3} \mathrm{~s}^{-1}$.

\section{Plasma cooling and electric field evolution}

The cooling process due to impurity radiation is described in a one dimensional cylindrical model [11] by the following energy balance equations

$$
\frac{3}{2} \frac{\partial\left(n_{\mathrm{e}} T_{\mathrm{e}}\right)}{\partial t}=\frac{3 n_{\mathrm{e}}}{2 r} \frac{\partial}{\partial r}\left(\chi r \frac{\partial T_{\mathrm{e}}}{\partial r}\right)+P_{\mathrm{OH}}-P_{\text {line }}-P_{\mathrm{br}}-P_{\text {ion }}+P_{\mathrm{c}}^{\mathrm{eD}}+P_{\mathrm{c}}^{\mathrm{eZ}},(7 \mathrm{a})
$$




$$
\begin{aligned}
& \frac{3}{2} \frac{\partial\left(n_{\mathrm{D}} T_{\mathrm{D}}\right)}{\partial t}=\frac{3 n_{\mathrm{D}}}{2 r} \frac{\partial}{\partial r}\left(\chi r \frac{\partial T_{\mathrm{D}}}{\partial r}\right)+P_{\mathrm{c}}^{\mathrm{De}}+P_{\mathrm{c}}^{\mathrm{DZ}}, \\
& \frac{3}{2} \frac{\partial\left(n_{\mathrm{Z}} T_{\mathrm{Z}}\right)}{\partial t}=\frac{3 n_{\mathrm{Z}}}{2 r} \frac{\partial}{\partial r}\left(\chi r \frac{\partial T_{\mathrm{Z}}}{\partial r}\right)+P_{\mathrm{c}}^{\mathrm{Ze}}+P_{\mathrm{c}}^{\mathrm{ZD}} .
\end{aligned}
$$

The electrons, deuterons and impurity ions are modelled separately, because of their different collision times. The different species are coupled with collisional energy exchange terms: $P_{\mathrm{c}}^{k l}=3 n_{k}\left(T_{l}-T_{k}\right) /\left(2 \tau_{k l}\right)$, where the heat exchange time is $\tau_{k l}=$ $3 \sqrt{2} \pi^{3 / 2} \epsilon_{0}^{2} m_{k} m_{l} /\left(n_{l} e^{4} Z_{k}^{2} Z_{l}^{2} \ln \Lambda\right)\left(T_{k} / m_{k}+T_{l} / m_{l}\right)^{3 / 2}$, and the subscripts $k$ and $l$ refer to electrons (e), deuterium ions (D) and impurities (Z). The heat diffusion coefficient is assumed to be constant $\chi=1 \mathrm{~m}^{2} \mathrm{~s}^{-1}$, unless otherwise indicated. The exact value of the heat diffusion coefficient in disruptions is not known so this is a free parameter in our model. The electron temperature changes due to Ohmic heating $\left(P_{\mathrm{OH}}=\sigma_{\|} E^{2}\right)$ and power losses caused by ionization $\left(P_{\text {ion }}\right)$, bremsstrahlung $\left(P_{\mathrm{br}}\right)$ and line radiation $\left(P_{\text {line }}=\sum_{i} n_{i} n_{\mathrm{e}} L_{i}\left(n_{\mathrm{e}}, T_{\mathrm{e}}\right)\right)$. Line radiation is the sum of the radiation for each charge state and the charge state densities $n_{i}$ evolve due to electron impact ionization and the radiative recombination. The radiation rates are extracted from the ADAS database [30]. Opacity effects could also be important, especially for low-Z impurities such as beryllium and carbon, as shown in [16], but for the noble gases (neon and argon) used in our study the difference in calculations with and without opacity effects is small. In the cases investigated in [16] it was shown that the neglecting the influence of the opacity may underestimate the temperature by a factor of about 2 , which in turn leads to an overestimation of the runaway current and a longer current decay time. Here we assume transparent plasmas, and therefore our results represent a worst-case scenario.

It is assumed that the final impurity profile $n_{\text {final }}(r)$ is proportional to the initial plasma density profile $n_{0}(r)=\left.n_{\mathrm{D}}(r)\right|_{t=0}=\left.n_{\mathrm{e}}(r)\right|_{t=0}$. The level of the final impurity density $n_{\text {final }}$ is varied between the different simulations, as well as the material $\left(n_{\text {final }}=n_{\text {Ar }}\right.$ or $\left.n_{\text {final }}=n_{\mathrm{Ne}}\right)$. In the beginning of the simulations we let the impurity density $n_{\mathrm{Z}}(r, t)$ increase exponentially to its final value according to $n_{\mathrm{Z}}=\left(1-e^{-t / t_{h}}\right) n_{\text {final }}$ on the time scale $t_{h}=1 \mathrm{~ms}$. This time scale was chosen because it is long enough to make the numerical calculations tractable but shorter than the relevant physical time scales. The time derivative of $n_{\mathrm{Z}}$ in Eq. (7c) soon gives a very small contribution to the rate of energy change for impurity ions in our calculations because the entering ions are very cold compared with the ions that are already present.

As the plasma cools and its conductivity drops $\left(\sigma_{\|} \sim T_{\mathrm{e}}^{3 / 2}\right)$, an electric field trying to maintain the current arises. The Maxwell equations and Ohm's law yield the following equation for the toroidal component of the electric field

$$
\frac{1}{r} \frac{\partial}{\partial r}\left(r \frac{\partial E}{\partial r}\right)=\mu_{0} \frac{\partial}{\partial t}\left(\sigma_{\|} E+n_{\text {run }} e c\right) .
$$

The total current is the sum of the Ohmic and runaway currents, assuming that the runaways travel with the speed of light. Equations (2), (7) and (8) are solved simultaneously to study plasma shutdown scenarios with neon and argon impurity injection. 

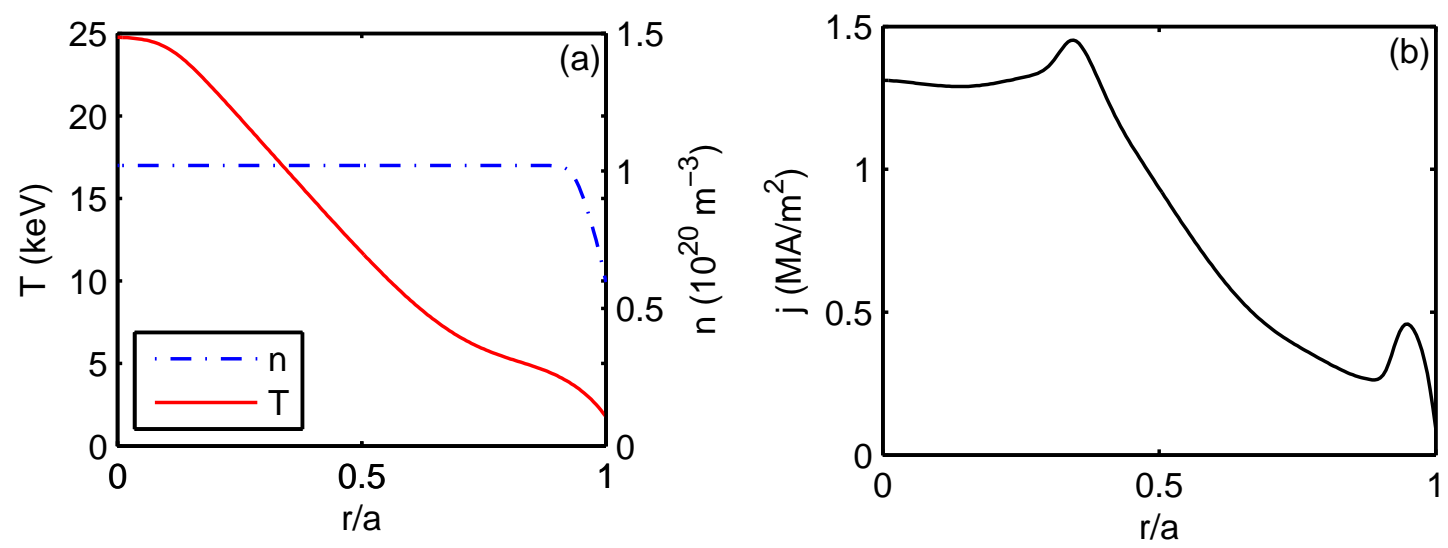

Figure 1: (a) Initial ITER density and temperature profiles as functions of normalized radius $r / a$. (b) Initial current profile.

\section{Results and discussion}

In our simulations we use a specific ITER-like scenario [31], obtained with the ASTRA code, with a plasma current of $15 \mathrm{MA}$, magnetic field $B=5.3 \mathrm{~T}$, major radius $R=6.2 \mathrm{~m}$, and mid-plane minor radius $2.0 \mathrm{~m}$. In our cylindrical model we use the radius $a=\sqrt{\kappa} 2.0 \mathrm{~m}$, where $\kappa=1.84$ is the elongation. Figure 1 shows the initial plasma temperature, density and current profiles. The central temperature and density are $T_{0}=24.7 \mathrm{keV}$ and $n_{e 0}=10^{20} \mathrm{~m}^{-3}$.

\subsection{Impurity injection}

To describe the physical processes, we first discuss how the temperature changes due to impurities, by examining two specific examples with $10 \%$ argon $\left(n_{\mathrm{Ar}}=0.1 n_{0}\right)$ and $50 \%$ neon $\left(n_{\mathrm{Ne}}=0.5 n_{0}\right)$ injection. The temperature evolution of the electrons, deuterium ions and argon ions at $r=0$ is shown in Fig. 2(a). The initial dramatic electron temperature-drop is mainly due to dilution caused by the large number of cold electrons that enter the plasma in the first milliseconds. During this time the thermal energy does not fall very much. Later, line-radiation and Bremsstrahlung induce a steady cooling of the plasma to a few eVs in about 115ms. Fig. 2(b) shows the time evolution of the radiation, Ohmic heating, ionization power densities and of the total thermal energy of the plasma. The Ohmic heating increases slowly as the temperature drops but it cannot compensate the radiation losses. After $115 \mathrm{~ms}$ a radiative collapse occurs and most of the remaining thermal energy is lost. Figures 2(c) and (d) show the same quantities for the injection of $50 \%$ neon. This choice of neon density was made so that the radiation collapse occurs at the same time as in the argon simulation. In the case of neon the background deuterium ions are cooled more rapidly, due to the larger number of injected impurities and the correspondingly larger collisional energy exchange. Figure 3 shows the time evolution of the number of ionized argon and neon atoms. The main difference is that due to the different ionization energies $\left(1.3 \mathrm{keV}\right.$ for $\mathrm{Ne}^{9+}$ and $4 \mathrm{keV}$ for $\mathrm{Ar}^{17+}$ ), 

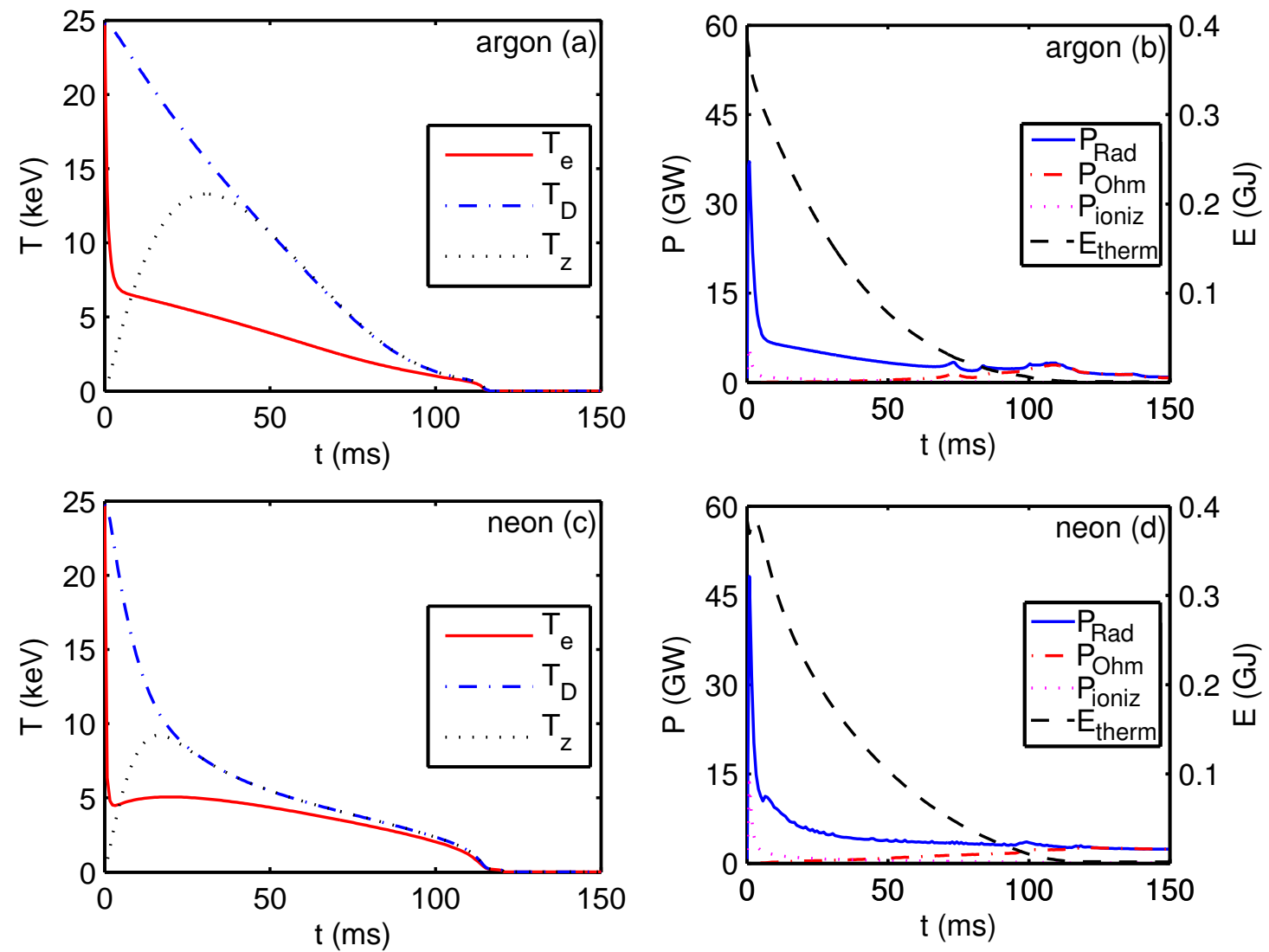

Figure 2: (a) Time evolution of the temperature of electrons, deuterium and argon ions at $r=0$ during injection of $10 \%$ argon. (b) Time evolution of radiation, Ohmic heating and ionization power (integrated over the whole volume) and the total thermal energy of the plasma. (c) Same as in (a) but for $50 \%$ neon. (d) Same as in (b) but for $50 \%$ neon.

most neon atoms are fully ionized. In spite of this, the radiation power density is almost the same both for argon and neon, since to obtain comparable cooling we injected more neon atoms and therefore have a correspondingly higher electron density. 

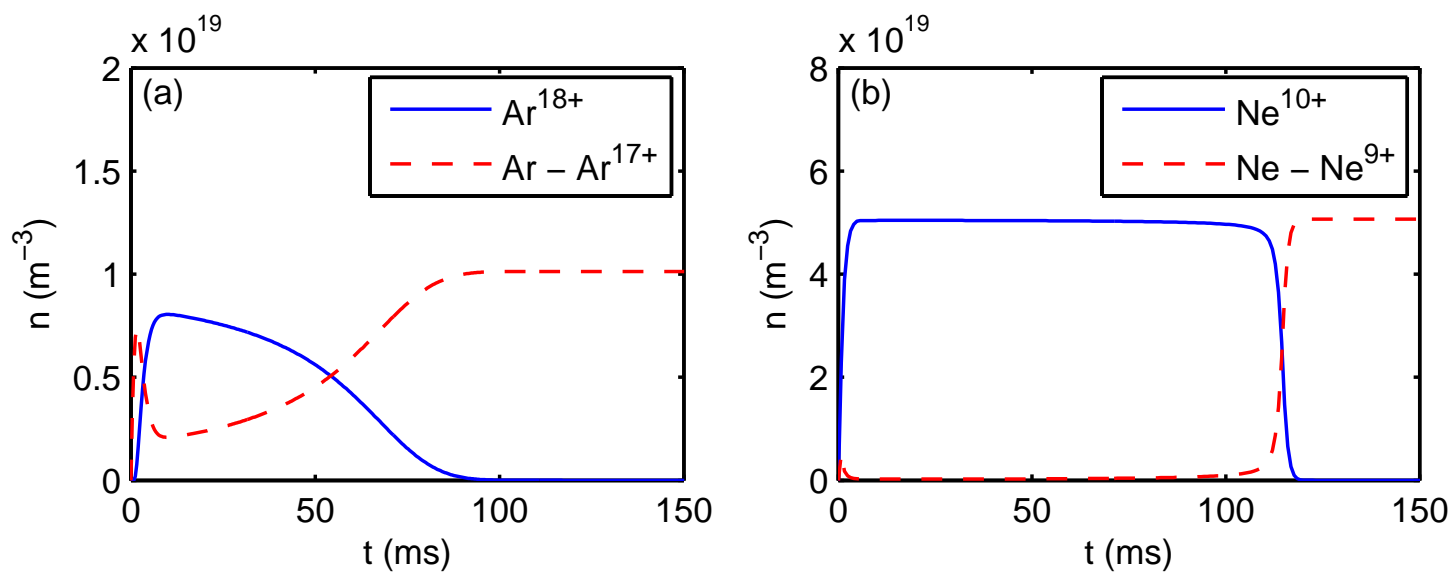

Figure 3: Time evolution at $r=0$ of the number of (a) fully ionized argon $\left(\mathrm{Ar}^{18+}\right)$ and all other argon atoms and ions, (b) fully ionized neon $\left(\mathrm{Ne}^{10+}\right)$ and all other neon atoms and ions. 

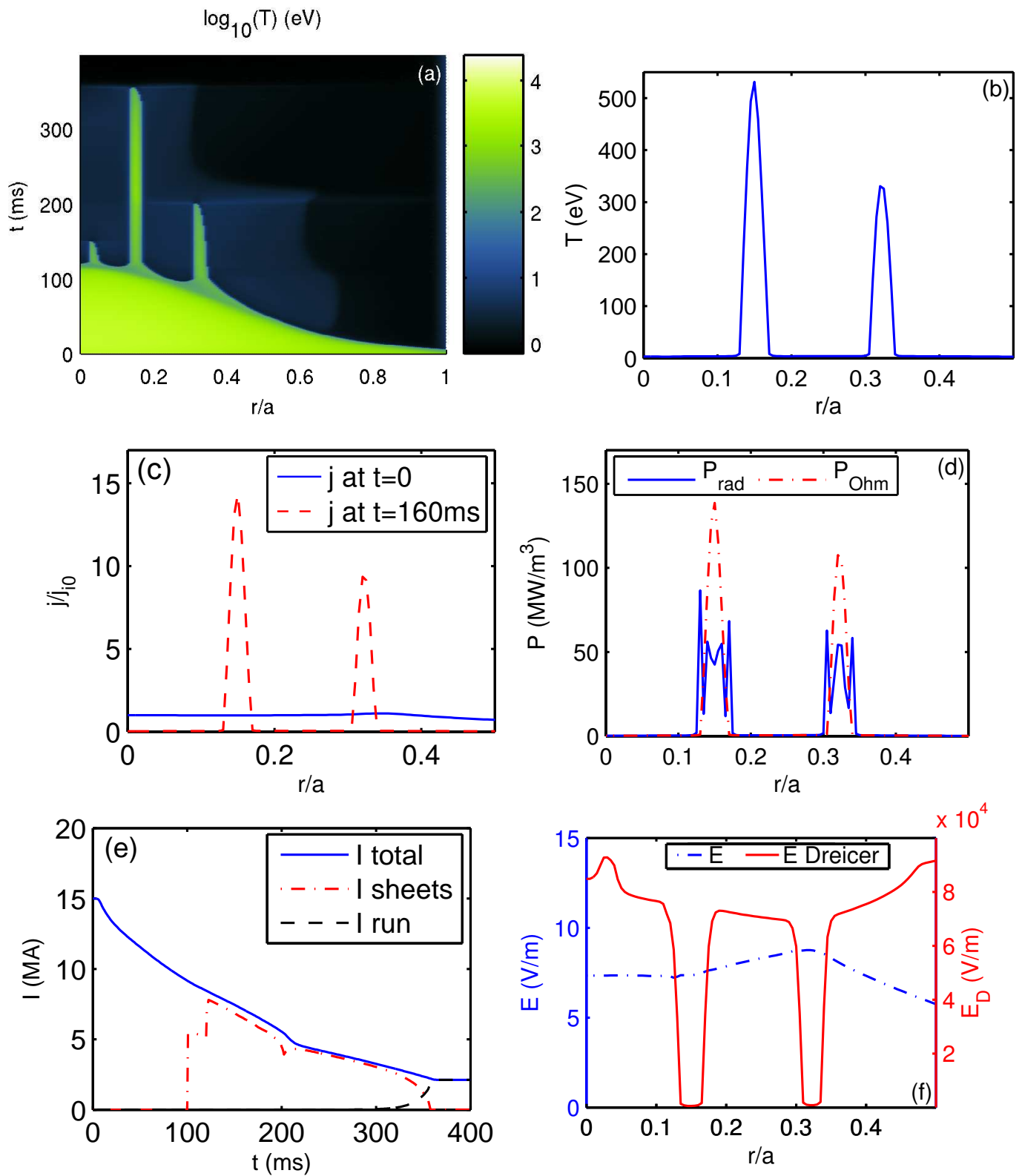

Figure 4: Current sheets are created after injection of neon into the plasma $\left(n_{\mathrm{Ne}} / n_{0}=0.5\right)$. (a) Electron temperature as a function of time and normalized radius. The other plots show plasma parameters as functions of normalized radius at $\mathrm{t}=160 \mathrm{~ms}$ : (b) electron temperature, (c) current density in the plasma, (d) radiation losses and Ohmic heating, (e) plasma current decay including the current driven inside the sheets (dash-dotted) and the runaway current, (f) electric field and Dreicer field (note the different scale for the Dreicer field $E_{D}$ ).

The temperature evolution in the whole plasma cross section is shown in Fig. 4(a). Due to a thermal instability resulting from a balance between heat diffusion, radiation and Ohmic heating, radially localised high temperature sheets are created. The physics behind the formation of these soliton-like structures was described in [2]. The temperature inside the sheets is a few hundred $\mathrm{eV}$ (Fig. 4(b)) and consequently the resistivity is much lower than in the surrounding plasma. As the high electric field 
from the surrounding cold region diffuses into the hot sheets it drives high current and keeps the region hot due to Ohmic heating. Figure 4(c) shows the current density of the plasma. The heat created in the center of the sheets diffuses outwards where it is radiated away (Fig. 4(d)). These current sheets exist for a long time and prolong the current quench (see Fig. 4(e)). In this work, in contrast to [2], we consider the Dreicer runaway generation process which is sensitive to the temperature. The Dreicer field is lower inside the sheets than outside (Fig. 4(f)). Furthermore the electric field changes only slightly with radius because there is enough time for the diffusion to smooth it out. The Dreicer generation strongly depends on the ratio of these two electric fields because $\dot{n}_{\text {run }}^{\text {Dreicer }} \sim \exp \left(-E_{\mathrm{D}} / E\right)$. As a result, Dreicer runaways are produced inside the sheets at a much higher rate than outside. Therefore, the peaked Ohmic current driven inside the sheets will be converted to an even more peaked runaway profile. The resulting runaway beams are sensitive to how the sheets are formed. A higher heat conductivity $(\chi)$ generally gives wider and fewer current sheets and if $\chi$ is high enough no sheets are created. Without current sheets the Dreicer generation becomes negligible compared to the other primary runaway mechanisms. In our simulations $\chi$ is chosen to be $1 \mathrm{~m}^{2} \mathrm{~s}^{-1}$ which is in the same order as the equilibrium value from the ASTRA code. If an MHD instability is present in the plasma a value of $100 \mathrm{~m}^{2} \mathrm{~s}^{-1}$ could be reasonable, in which case the current sheets disappear. The influence of different heat conductivity values are further detailed in Sec. 4.4.

\subsection{Density scan}

Simulations with different argon concentration were performed, taking all runaway generation processes (Dreicer, hot-tail, $\gamma$-ray and avalanche mechanism) into account and neglecting the losses. In most cases current sheets are created and the Dreicer generation inside the sheets is the strongest primary runaway generation mechanism. At high impurity concentration the thermal quench is short, so the hot tail generation becomes strong. To compare the different primary runaway generation processes we can integrate the terms on the right hand side in in Eq. (2) separately. The result is shown in Fig. 5. The $\gamma$ term is weak compared to the other primary mechanisms in these scenarios. For argon content $n_{\mathrm{Ar}} / n_{0}>0.9$ the hot-tail generation is the dominant seed mechanism. This creates a broad runaway current profile which is later amplified by the avalanche mechanism, see Fig. 6(a). For lower argon concentration the Dreicer generation inside the sheets dominates, which leads to spikes in the runaway current profile as shown in Fig. 6(b) for $n_{\mathrm{Ar}} / n_{0}=0.1$.

Simulations with different concentrations of neon and argon were performed to determine the thermal and current quench times and the total runaway current generated. These simulations were also done without runaway losses. Figure 7(a) shows the thermal and current quench times, and the generated runaway current is presented in Fig. $7(\mathrm{~b})$. The displayed current quench time $\left(\tau_{\mathrm{CQ}}\right)$ is defined as the time in which the Ohmic current drops to $1 / e$ of its initial value, and similarly the thermal quench 


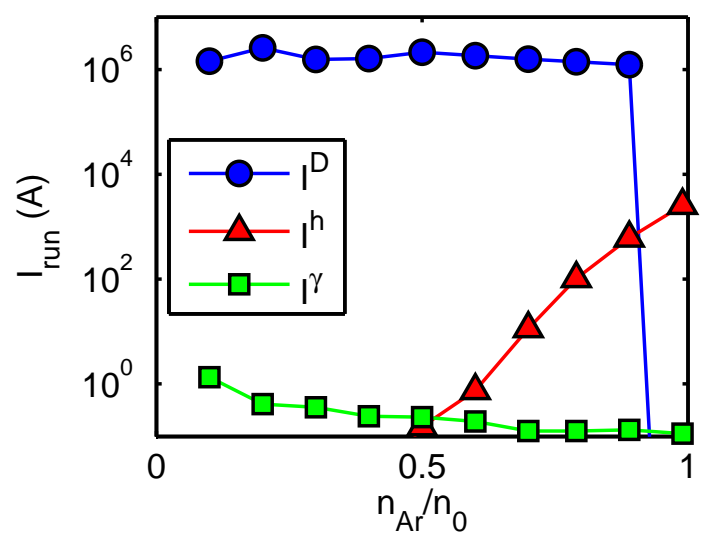

Figure 5: Comparison of primary runaway sources at the time when the Ohmic current disappears from the plasma (the beginning of the runaway plateau). $I^{\mathrm{D}}=e c \int \dot{n}_{\text {run }}^{\text {Dreicer }} d \mathrm{~A} d \mathrm{t}$, $I^{\mathrm{h}}=e c \int \dot{n}_{\mathrm{run}}^{\text {hot-tail }} d \mathrm{~A} d \mathrm{t}, I^{\gamma}=e c \int \dot{n}_{\mathrm{run}}^{\gamma} d \mathrm{~A} d \mathrm{t}$.
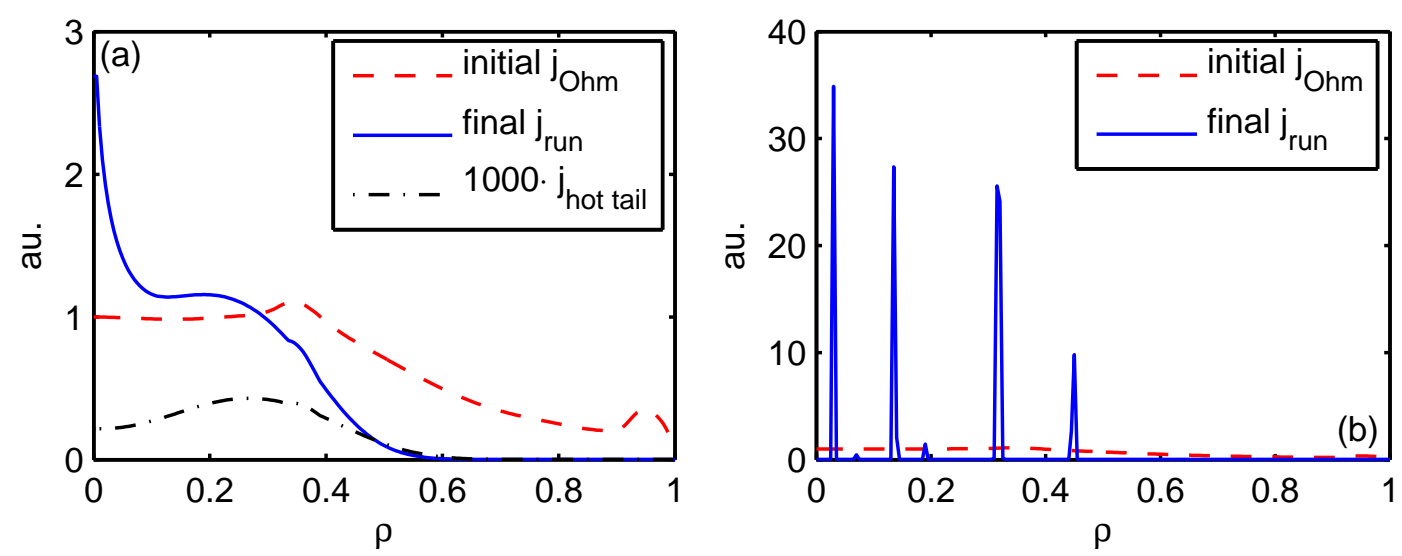

Figure 6: Comparison of Dreicer and hot-tail runaway current at the end of current quench. Initial current density and final runaway current density from a simulation with (a) $n_{\mathrm{Ar}} / n_{0}=$ 1.0 and (b) $n_{\mathrm{Ar}} / n_{0}=0.1$. The hot-tail seed population is magnified with a factor of 1000 for better visibility.

time $\left(\tau_{\mathrm{TQ}}\right)$ is the time needed for the total thermal energy to drop to $1 / e$ of the initial thermal energy. In case of argon, all simulations result in a significant runaway current. Increasing the argon content increases the electron density, and therefore slightly reduces the final runaway current. The thermal quench time decreases if more argon is injected and the hot-tail seed therefore becomes strong for densities $n_{\mathrm{Ar}} / n_{0} \gtrsim 50 \%$. In the case of neon injection, if $n_{\mathrm{Ne}} / n_{0}<2$ the $\gamma$-ray Compton scattering seed is usually stronger than the Dreicer generation and therefore the runaway current is almost independent of the amount of injected neon. For $n_{\mathrm{Ne}} / n_{0}=2$, the Dreicer generation is strong in the high temperature sheets and this creates a peak in the total runaway current. For higher neon density the hot-tail generated runaway seed becomes also important.

There is a significant difference in thermal and current quench times between neon and argon. Clearly argon is more efficient in cooling the plasma, but this efficient cooling 

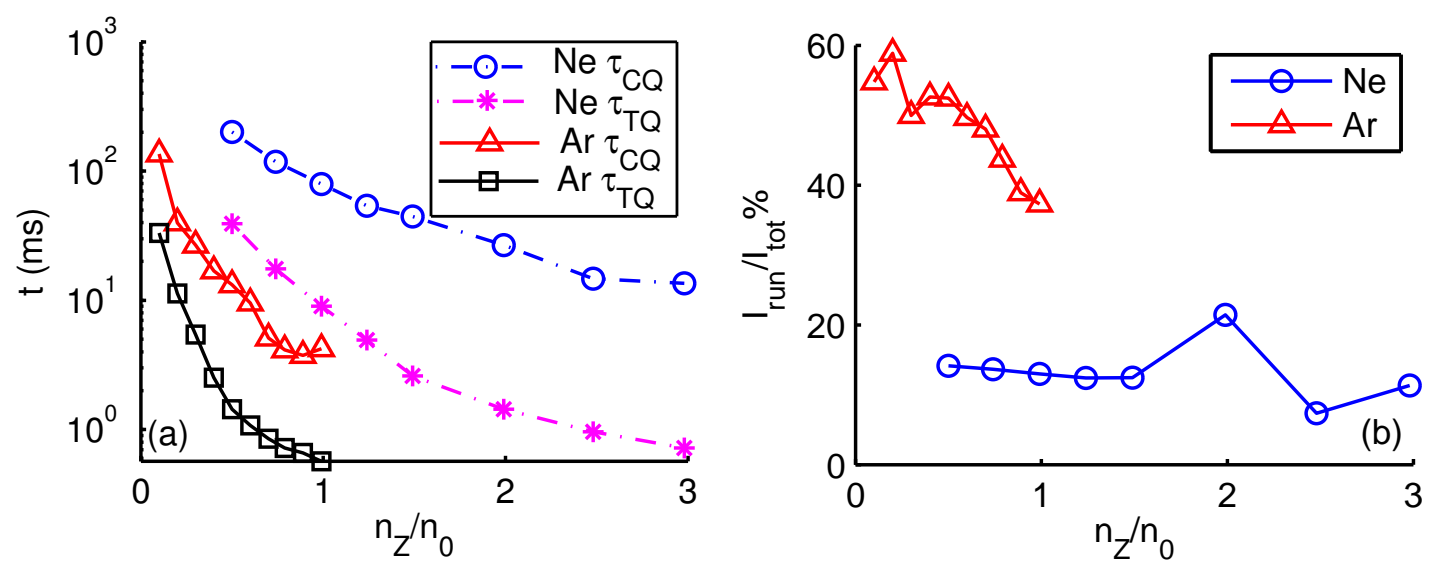

Figure 7: (a) Thermal quench time $\left(\tau_{\mathrm{TQ}}\right)$ and current quench time $\left(\tau_{\mathrm{CQ}}\right)$ after injection of different amounts of neon and argon into the plasma. (b) Generated runaway current.

and the short thermal quench time also has the effect that the RE current is much higher for argon than for neon.

To reduce runaway generation a mixture of deuterium and impurities could be injected into the plasma. With deuterium the electron density can be increased without significantly decreasing the thermal quench time. Figure 8 compares the thermal quench time, current quench time and runaway current for different amounts of argon with and without massive deuterium injection $\left(n_{\mathrm{D}} / n_{0}=10\right)$. The thermal quench becomes somewhat shorter in the cases where large amounts of cold deuterium particles are introduced, but it does not change significantly. The main difference when the extra deuterium is added is that the runaway current is reduced for low argon density cases. The reason is that the high electron density caused by the large amount of deuterium decreases the Dreicer generation, as can be seen in Fig. 9. This figure presents the final Dreicer, hot-tail and $\gamma$-ray Compton scattering runaway seed currents generated during simulations with $n_{\mathrm{D}} / n_{0}=10$ and different argon contents. The Dreicer generation is smaller compared with the case without the added deuterium in Fig. 5. For argon densities in the range of $0.2 n_{0}-0.5 n_{0}$ the Dreicer generation is still active in the high temperature sheets. At other densities the sheets do not form and the Dreicer generation is negligible. The extra deuterium does not affect the $\gamma$-ray Compton scattering generation. The hot-tail and avalanche generation are also more difficult to reduce [19]. Even with a ten-fold deuterium density increase the avalanche generation is not suppressed, as seen in Fig. 8(b). If the argon concentration is too large, the hot-tail process provides a seed which is amplified to a high final runaway current. On the other hand, at lower densities the $\gamma$-ray Compton scattering seed is also amplified to a considerable final runaway current.

In order to suppress the avalanche in these scenarios, the critical electric field should be larger than the actual electric field, and to accomplish this we would need a more than hundred-fold density increase. In reality, such a large amount of gas would put a serious load on the vacuum pumps. Instead of further increasing the deuterium density, 
in the following we will examine shutdown scenarios with runaway losses that could counteract the avalanche mechanism.
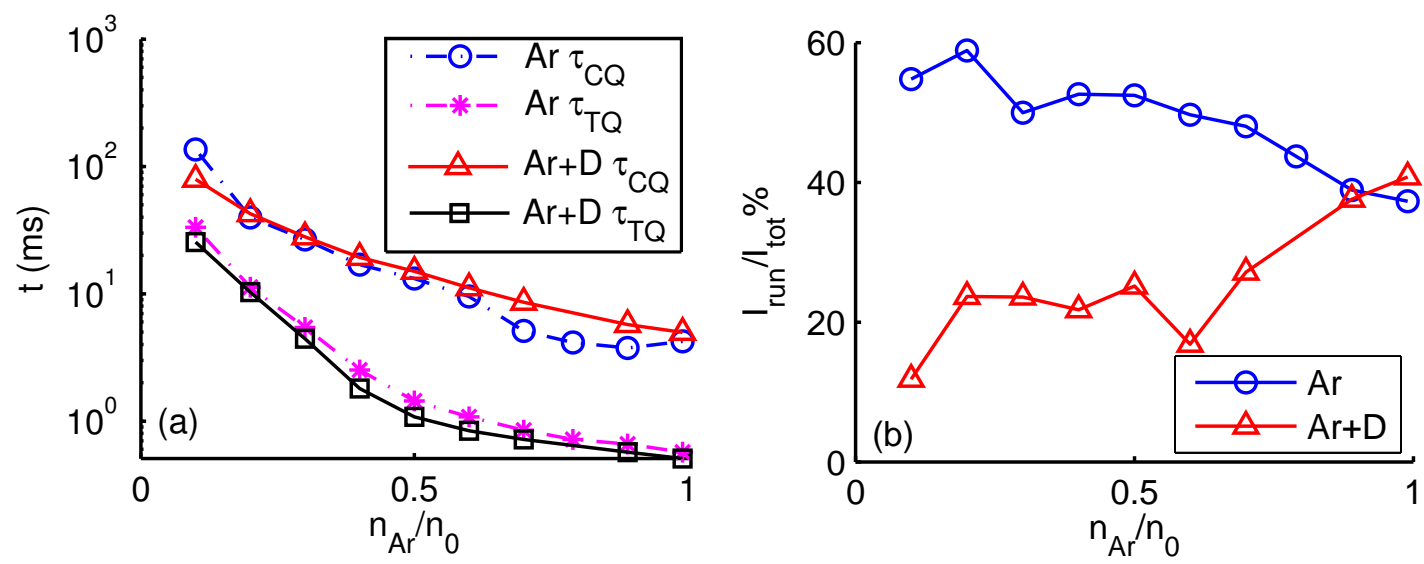

Figure 8: (a) Thermal quench time and current quench time after injection of different amounts of argon with and without deuterium $\left(n_{\mathrm{D}} / n_{0}=10,+\mathrm{D}\right.$ in the legend) into the plasma. (b) The corresponding runaway current.

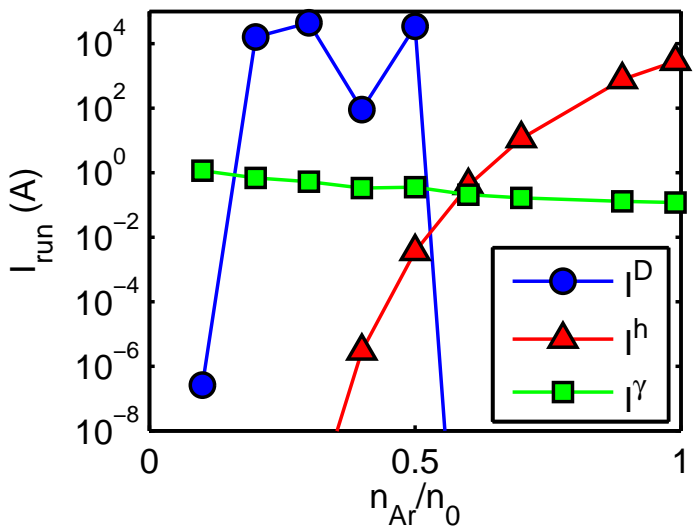

Figure 9: Comparison of accumulated primary runaway sources at the time when the Ohmic current decreases to zero in simulations with $n_{\mathrm{D}} / n_{0}=10$ and different argon concentrations. The currents from Dreicer $\left(\mathrm{I}^{D}\right)$, hot-tail $\left(\mathrm{I}^{h}\right)$, and $\gamma\left(\mathrm{I}^{\gamma}\right)$ generation processes are defined the same way as in Fig. 5. The Dreicer generation is reduced by the density increase caused by the extra deuterium. 


\subsection{Runaway losses}

Runaway electron diffusion due to magnetic perturbations can reduce the runaway density. In this section the effect of the magnetic perturbation is studied for an argon scenario with $n_{\mathrm{Ar}} / n_{0}=1.0$ and no added deuterium. In this scenario hot-tail generation creates a runaway current which is amplified to a strong runaway beam if we do not have losses. Runaway current fractions from simulations with different magnetic perturbation levels are presented in Fig. 10, which shows the maximum runaway current, because the runaway current changes with time. The time evolution of the runaway current and the total current is shown in Fig. 11 for four different values of $\delta B / B$.

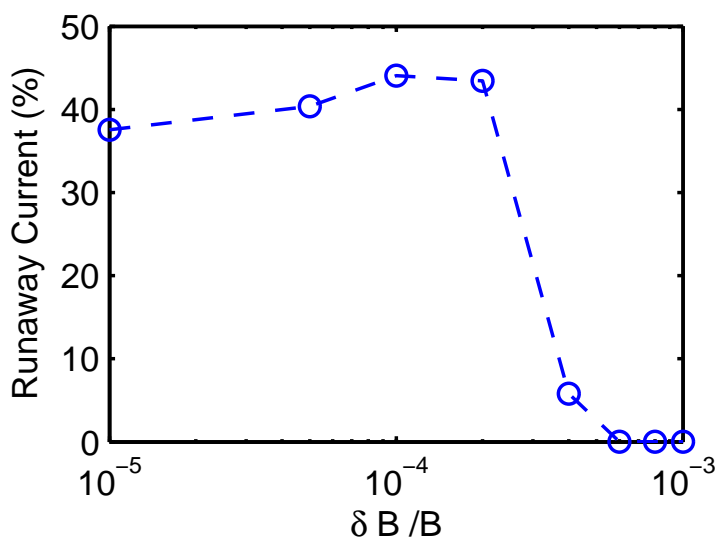

Figure 10: Runaway fraction of total current as a function of normalized magnetic field perturbations at injection of $\operatorname{argon}\left(n_{\mathrm{Ar}} / n_{0}=1.0\right)$.

Without runaway losses the simulation ends with high runaway current (Fig. 11(a)), because there is a strong initial seed runaway profile due to the hot-tail effect. When the magnetic perturbation level is increased up to $\delta B / B \sim 10^{-4}$, radial diffusion makes this seed profile broader (see Fig. 12), which allows the avalanche mechanism to create an even higher final runaway current. For $\delta B / B=2 \cdot 10^{-4}$ (Fig. 11(b)) there are significant runaway losses, but in the first few ms when the electric field is very high it cannot yet counteract the avalanche mechanism. With high magnetic perturbation $\left(\delta B / B \geq 8 \cdot 10^{-4}\right.$, Fig. $\left.11(\mathrm{~d})\right)$ the runaway loss rate is higher than the avalanche rate and the runaway electrons generated by the hot-tail process will diffuse out of the plasma before they can form a strong runaway beam, as predicted in [23]. The observed level of magnetic perturbations in existing large tokamaks (such as JET) is larger than $10^{-3}$ at the stage of the current quench [32], and if the same level of magnetic perturbation will be present in ITER, it would be sufficient to scatter the runaway beam. 

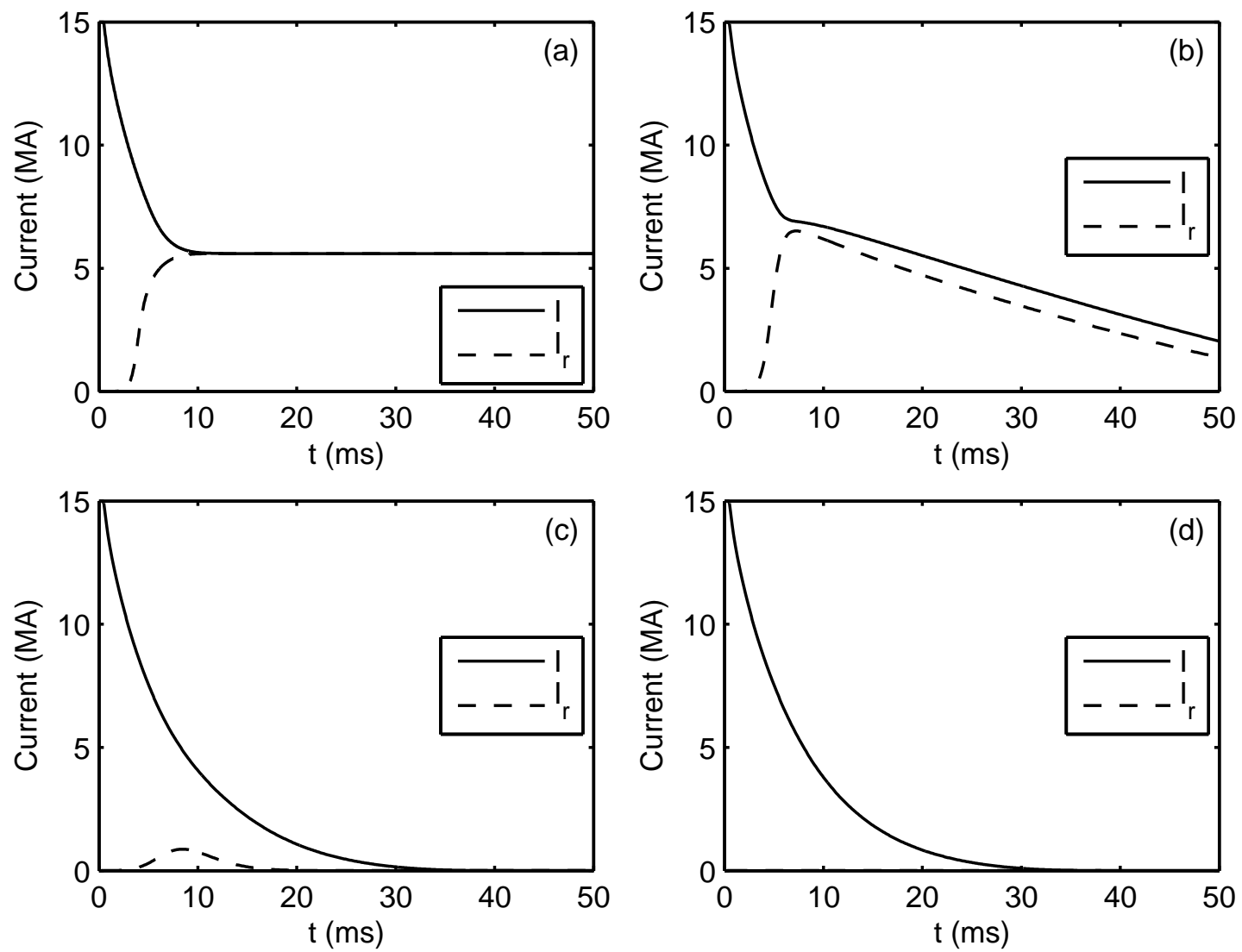

Figure 11: Time evolution of the total current and runaway current for different normalized magnetic field perturbations and injection of $n_{\mathrm{Ar}} / n_{0}=1.0$. (a) $\delta B / B=0$, (b) $\delta B / B=$ $2 \times 10^{-4}$, (c) $\delta B / B=4 \times 10^{-4}$, (d) $\delta B / B=8 \times 10^{-4}$.

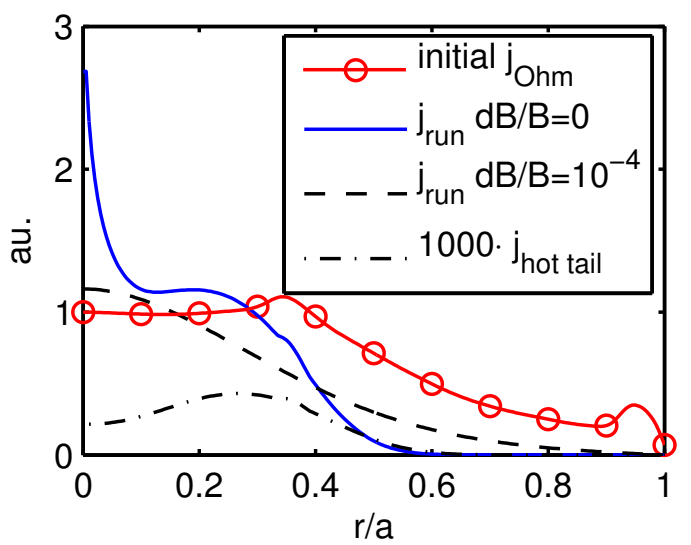

Figure 12: Initial current density and final runaway current density as a function of normalized radius from a simulation with $\delta B / B=0$ and $\delta B / B=10^{-4}$. The hot-tail seed population is also shown and it is magnified with a factor of 1000 for better visibility. Note that the runaway current density profile gets broader when $\delta B / B$ is larger. 


\subsection{Sensitivity to the impurity profile and heat conduction coefficient}

Various tests were performed to investigate the effect of our assumptions. First of all we tested the sensitivity of our results to the predescribed impurity density profile. During a controlled shutdown the aim is to achieve efficient cooling all along the plasma radius, which means that the impurity density has to be above some minimum level throughout the whole plasma. The parts of the plasma where this minimum level is not reached will remain hot and prolong the thermal and current quench of the plasma. The electric field will diffuse into these hot regions, thereby strengthening the runaway generation, and furthermore, the effect of runaway generation reduction by electron density increase will be weaker. As long as the impurity content is sufficiently high throughout the plasma, the details of the radial impurity density profile does not not have a decisive influence on the runaway generation. To illustrate this, simulations were performed with the four different impurity profiles in Fig. 13. The temperature profile evolution is different for

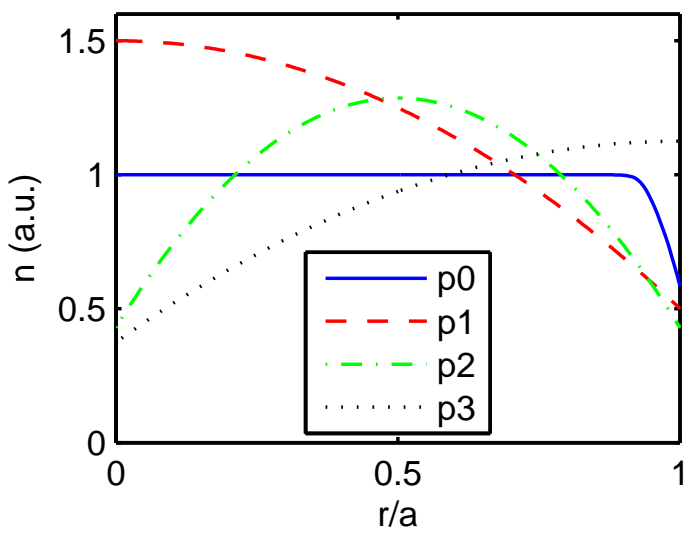

Figure 13: Different impurity profiles $p_{0} \sim n_{0}, p_{1} \sim 1.5-x^{2}, p_{2} \sim 1.5-4(x-0.5)^{2}$, $p_{3} \sim 1.5-(x-1)^{2}$. The profiles are normalised so that the total impurity particle number is the same.

each impurity profile as it strongly depends on the local impurity/background plasma density ratio. However, as Fig. 14(a) shows, the thermal quench times are similar in all cases. If we compare the current quench times (Fig. 14(b)) we see that the more impurities there are in the center the faster the quench will be. Fig. 14(c) shows that all the profiles result in a significant runaway current. There is \pm 15 percentage point variation in the final runaway current depending on the impurity density profile, but since all cases result in a large runaway current and the differences in thermal and current quench times are small, the main conclusions of the paper do not depend on the choice of exact impurity density profile.

To mimic the penetration of material from the edge to the centre of the plasma, simulations were set up with a spatially dependent time delay before the density begins to increase from zero to its final value,

$$
n_{Z}(r, t)= \begin{cases}n_{\text {final }}(r)\left(1-e^{-(t-\operatorname{delay}(r)) / t_{h}}\right) & \text { if } t>\operatorname{delay}(r) \\ 0 & \text { otherwise }\end{cases}
$$



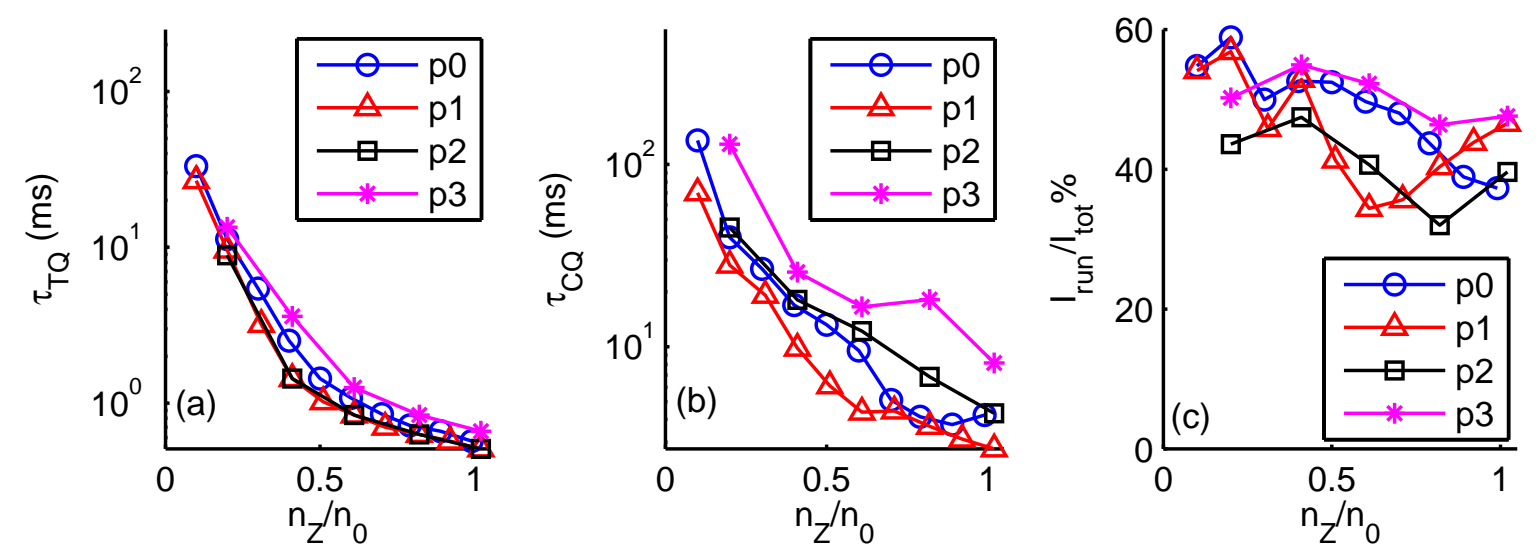

Figure 14: Simulation results for the impurity profiles in Fig. 13. (a) Thermal quench time, (b) current quench time and (c) generated runaway current.

where $\operatorname{delay}(r)=(a-r) / v$. The delay function was chosen as the time it would take a $v=600 \mathrm{~m} / \mathrm{s}$ pellet to reach point $r$. The delay in the center is around $4.5 \mathrm{~ms}$. The radial impurity profile was similar to the background density profile shape $\left(n_{\text {final }}(r) \sim n_{0}(r)\right)$ and the temperature evolution of the background plasma was compared to the case where the time for impurity penetration was neglected. Figure 15 shows the temperature profile evolution. The cooling process does not change qualitatively when the delay is introduced. Besides the 4-5 ms offset caused by the delay the final thermal and current quench times does not change considerably, as can be seen in Fig. 16.
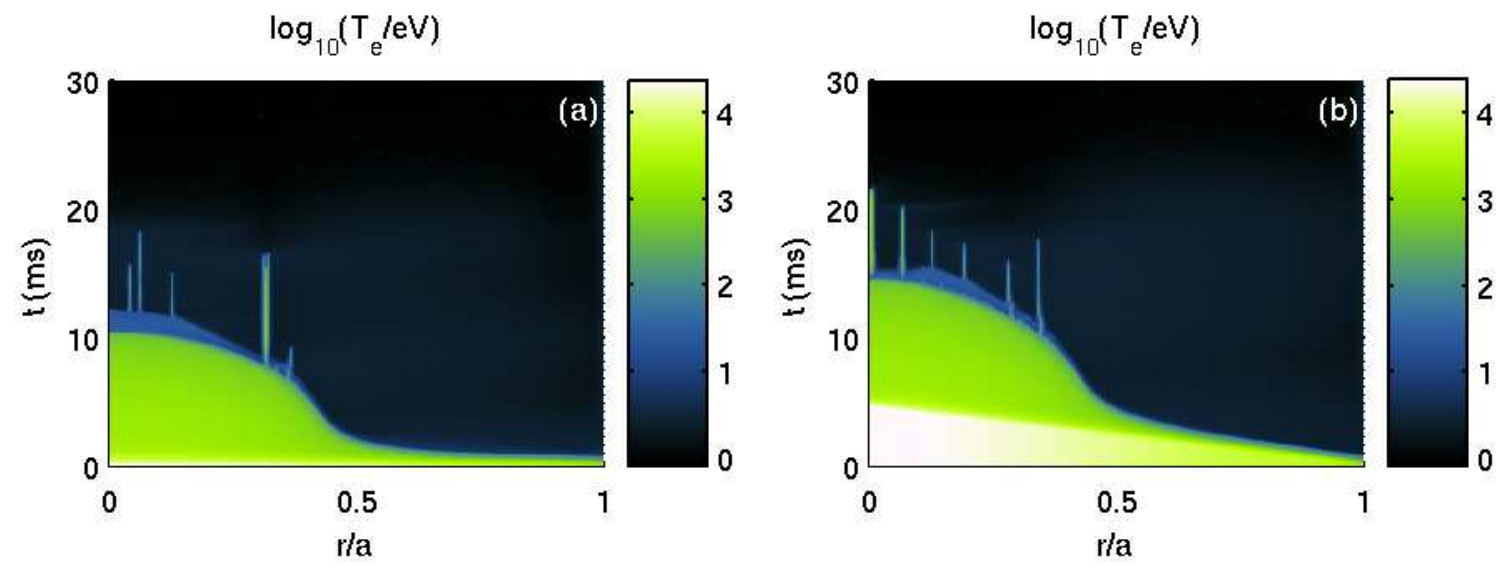

Figure 15: Temperature profile evolution (a) without delay and (b) with delay (50\% argon).

To study the effect of different heat conduction coefficients, argon scenarios were investigated with $\chi=5 \mathrm{~m}^{2} / \mathrm{s}$ and $\chi=10 \mathrm{~m}^{2} / \mathrm{s}$. The results (Fig. 17) indicate that the thermal and current quench times do not change significantly. As previously mentioned, the sheet structures become radially broader with higher heat conduction. The temperature inside such thick sheets is also lower, and since the Dreicer generation is sensitive to the temperature, the runaway current is found to decrease with increasing 

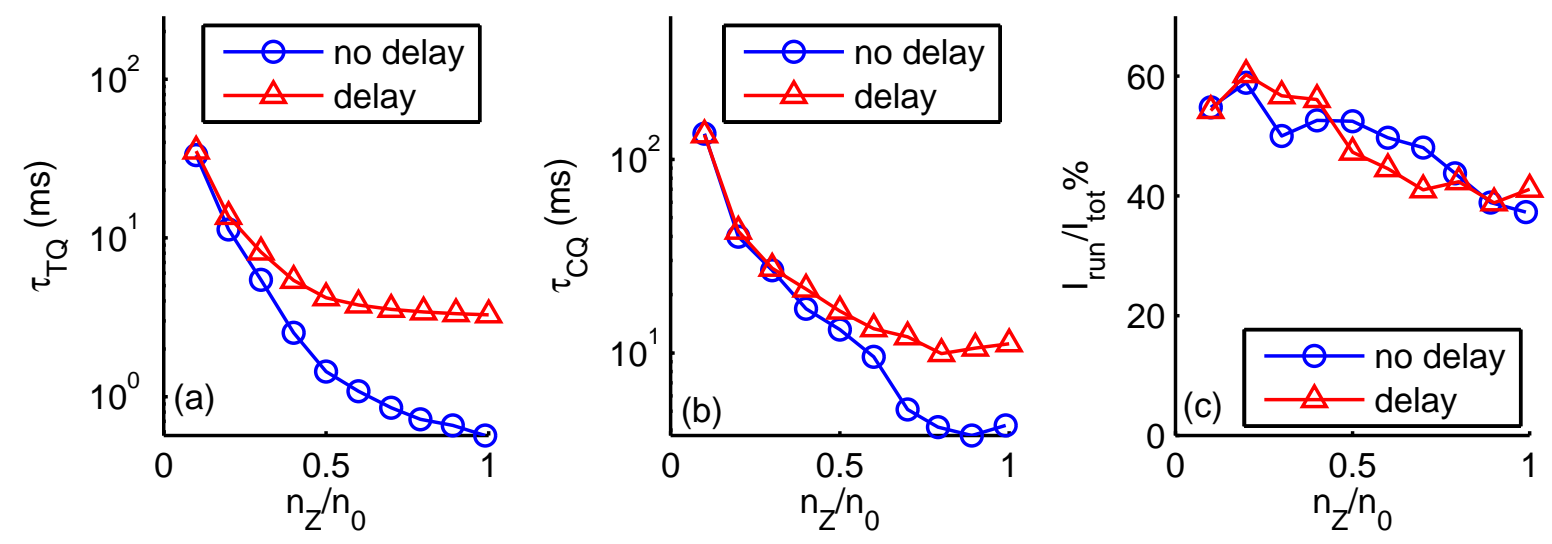

Figure 16: (a) Thermal quench time, (b) current quench time and (c) generated runaway current with and without delay. The quench times are in all cases the time interval from the beginning of the simulation to the time when the $1 / e$ level is reached. The longer quench times for the delay cases are caused by the $4-5$ ms delay in the central plasma.

$\chi$ at low argon densities. At high argon densities $\left(\mathrm{n}_{\mathrm{Ar}} / n_{0} \geq 0.7\right)$ there is a strong hot-tail seed and it is only slightly influenced by the change in the heat conduction. The final runaway current changes only moderately in these cases.
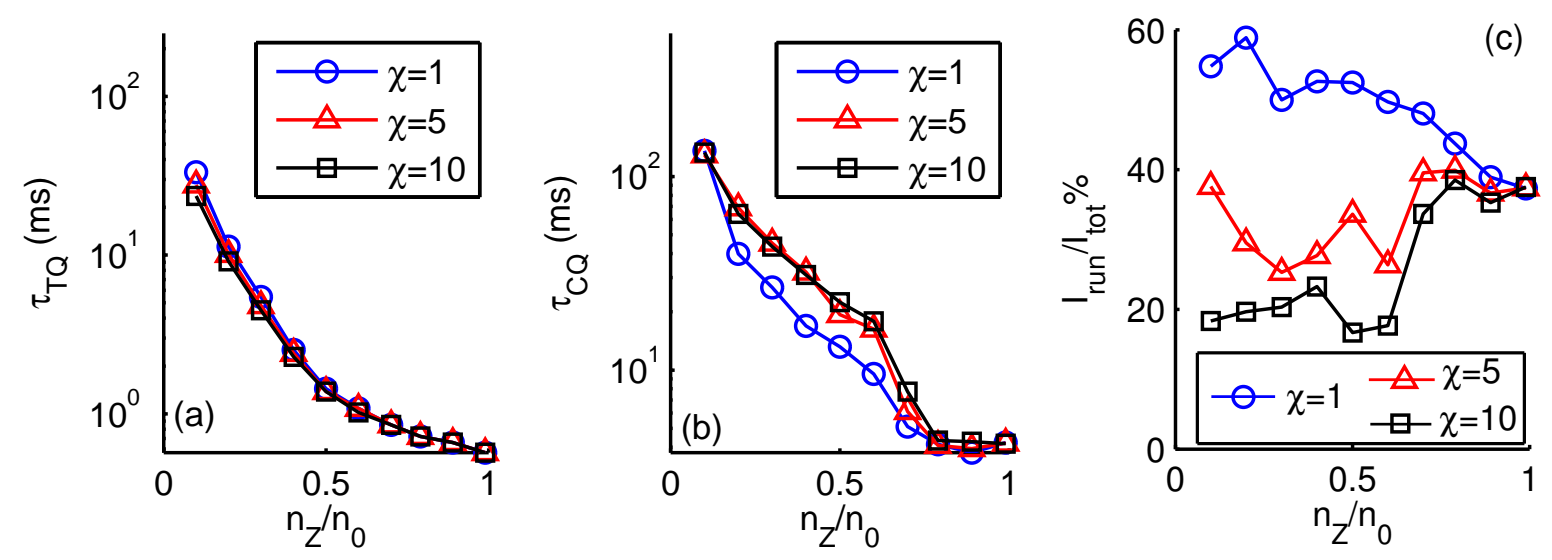

Figure 17: (a) Thermal quench time, (b) current quench times and (c) runaway current as a function of argon density using different heat conduction coefficients.

\section{Conclusions}

Fast plasma shutdown scenarios with neon and argon impurities were simulated for ITER-like parameters, with a 1D code solving the coupled equations of electric field diffusion, runaway generation and energy balance. For simplicity, the complicated physics of how the impurities are transported into the plasma was not modelled, but a predescribed impurity profile was used. The change of the plasma temperature is calculated taking radiation, Ohmic heating, collisions between species and heat diffusion 
into account. Dreicer, hot-tail, $\gamma$-ray Compton scattering and avalanche runaway electron generation during the current quench and the evolution of the electric field are determined self-consistently. Runaway losses were also calculated with a simple model for diffusion due to magnetic perturbations.

Our results indicate that injection of high-Z impurities, such as argon or neon is very efficient in cooling the plasma, but can lead to high runaway current. Primary runaway electrons are generated by the hot-tail process when $n_{A r} \sim n_{0}$ ( or $n_{N e}>2 n_{0}$ ) or by the Dreicer process at lower impurity densities. The avalanche generation is not suppressed for the studied impurity concentrations $\left(n_{Z} / n_{0} \sim O(1)\right)$ and it further amplifies the runaway current. Long-lived radially localised sheets of high temperature can be formed after the thermal quench and this influences the Dreicer generation. The Dreicer generation is strong inside these sheets and it can create runaway beams with high current. When the impurity content is high, sheets do not form, and the Dreicer generation is therefore not significant. Instead, the large amount of impurities make the thermal quench time so short that hot-tail generation becomes the dominant runaway seed mechanism.

The Dreicer seed can be suppressed if we increase the plasma density by using a mixture of deuterium and neon or argon, but the avalanche mechanism is not suppressed. If we have other strong primary generation processes, such as hot-tail or $\gamma$-ray Compton scattering, then we have to rely on runaway loss mechanisms that can counteract the avalanche. A simple model for losses due to runaway diffusion was used to show that for $n_{\mathrm{Ar}} \sim n_{0}$, a magnetic perturbation level $\delta B / B=10^{-3}$ is sufficient to stop runaway avalanching.

\section{Acknowledgements}

This work was funded by the European Communities under Association Contract between EURATOM, Germany, HAS and Vetenskapsrådet. The views and opinions expressed herein do not necessarily reflect those of the European Commission. The authors would like to thank P Helander for fruitful discussions.

[1] Shimada M, Campbell D, Fujiwara V M M, Kirneva N, Lackner K, Nagami M, Pustovitov V, Uckan N and Wesley J 2007 Nucl. Fusion 47 S01

[2] Putvinski S, Fujisawa N, Post D, Putvinskaya N, Rosenbluth M N and Wesley J 1997 J. Nucl. Mater. 241-243 316-321

[3] Putvinski S, Barabaschi P, Fujisawa N, Putvinskaya N, Rosenbluth M N and Wesley J 1997 Plasma. Phys. Control. Fusion 39 B157-B171

[4] Hollmann E M, Jernigan T, Groth M, Whyte D and Gray D 2008 Nucl. Fusion 48115007

[5] Pautasso G, Fuchs C, Gruber O, Maggi C, Maraschek M, Pütterich T, Rohde V, Wittmann C, Wolfrum E, Cierpka P, Beck M and the ASDEX Upgrade Team 2007 Nucl. Fusion 47 900-913

[6] Bakhtiari M, Tamai H, Kawano Y, Kramer G, Isayama A, Nakano T, Kamiya Y, Yoshino R, Miura Y, Kusama Y and Nishida Y 2005 Nucl. Fusion 45318

[7] Whyte D G, Jernigan T, Humphreys D A, Hyatt A W, Lasnier C J, Parks P B, Evans T E, Taylor P L, Kellman A G, Gray D S and Hollman E M 2003 J. Nucl. Mater. 313-316 1239-1246 
[8] Pautasso G, Buchl K, Fuchs J, Gruber O, Herrmann A, Lackner K, Lang P T, Mast K F, Ulrich M, Zohm H and the ASDEX Upgrade Team 1996 Nucl. Fusion 361291

[9] Taylor P L, Kellman A G, Evans T E, Gray D S, Humphreys D A, Hyatt A W, Jernigan T C, Lee R L, Leuer J A, Luckhardt S C, Parks P B, Schaffer M J, Whyte D G and Zhang J 1999 Phys. Plasmas 61872

[10] Yoshino R, Kondoh T, Neyatani Y, Itami K, Kawano Y and Isei N 1997 Plasma Phys. Control. Fusion 39 313-332

[11] Gál K, Fehér T, Smith H, Fülöp T and Helander P 2008 Plasma Phys. Control. Fusion 50055006

[12] Jardin S C, Schmidt G L, Fredrickson E D, Hill K W, Hyun J, Merrill B J and Sayer R 2000 Nucl. Fusion 40923

[13] Harvey R W, Chan V S, Chiu S C, Evans T E, Rosenbluth M N and Whyte D G 2000 Phys. Plasmas 74590

[14] Izzo V A, Whyte D G, Granetz R S, Parks P B, Hollmann E M, Lao L L and Wesley J C 2008 Phys. Plasmas 15056109

[15] Pautasso G, Coster D, Bonnin X, Eich T, Fuchs J, Kurzan B, McCormick K, Reiter B, Rohde V and the ASDEX Upgrade Team 2008 Proc. 22nd Int. Conf. on Fusion Energy (Geneva, Switzerland, 2008) (Vienna: IAEA) pp CD-ROM file IAEA-CN-165/EX/P9-1

[16] Lukash V E, Mineev A B and Morozov D K 2007 Nucl. Fusion 47 1476-1484

[17] Eriksson L G, Helander P, Andersson F, Anderson D and Lisak M 2004 Phys. Review Letters 92 205004-1

[18] Smith H, Helander P, Eriksson L G, Anderson D, Lisak M and Andersson F 2006 Phys. Plasmas 13102502

[19] Smith H M and Verwichte E 2008 Phys. Plasmas 15072502

[20] Smith H, Fehér T, Fülöp T, Gál K and Verwichte E 2009 Plasma Phys. Control. Fusion 51124008

[21] Eriksson L G and Helander P 2003 Comp. Phys. Comm. 154175

[22] Rechester A B and Rosenbluth M N 1978 Phys. Review Letters 4038

[23] Helander P, Eriksson L G and Andersson F 2000 Phys. Plasmas 74106

[24] Fülöp T, Smith H M and Pokol G 2009 Phys. Plasmas 16022502

[25] Kruskal M D and Bernstein I B 1962 PPPL Report 174 MATT-Q-20

[26] Smith H, Helander P, Eriksson L G and Fülöp T 2005 Phys. Plasmas 12122505

[27] Wesson J 1997 Tokamaks (Oxford University Press) chap 14.5 2nd ed

[28] Rosenbluth M N and Putvinski S V 1997 Nucl. Fusion 371355

[29] Iida H, Khripunov V, Petrizzi L and Federici G 2004 Nuclear Analysis Report

[30] Smith H P 2004 The ADAS User Manual, version 2.6

[31] Polevoi A private communication

[32] Wesson J A, Gill R D, Hugon M, Schüller F C, Snipes J A, Ward D J, Bartlett D V, Campbell D J, Duperrex P A, Edwards A W, Granetz R S, Gottardi N A O, Hender T C, Lazzaro E, Lomas P J, Cardozo N L, Mast K F, Nave M F F, Salmon N A, Smeulders P, Thomas P R, Tubbing B J D, Turner M F and Weller A 1989 Nucl. Fusion 29 641-666 